${ }^{1}$ Национальный медицинский исследовательский центр эндокринологии, Москва, Россия

${ }^{2}$ Такеда Фармасьютикалс, Москва, Россия

ОБОСНОВАНИЕ. В связи с полученными данными о влиянии алоглиптина на липидный профиль, массу тела и артериальное давление (АД) пациентов был проведен дополнительный анализ результатов исследования ENTIRE, завершенного в РФ в 2018 г.

ЦЕЛЬ. Оценить динамику $\mathrm{HbA}_{1 c^{\prime}}$ массы тела, показателей липидного спектра, АД и охарактеризовать профиль пациента, получившего максимальную клиническую пользу на фоне терапии алоглиптином в ходе исследования ENTIRE.

МЕтоды. Проспективное неинтервенционное наблюдательное исследование, в которое включались пациенты в возрасте 18 лет и старше с впервые диагностированным сахарным диабетом 2 типа (СД2) или пациенты с СД2, не достигшие целевых показателей гликемии на фоне предшествующей терапии.

РЕзУЛЬтАты. Снижение гликированного гемоглобина ( $\left.\mathrm{HbA}_{1 c}\right)$ более чем на 0,5\% выявлено у 73,5\% пациентов. Наиболее выраженное абсолютное снижение $\mathrm{HbA}_{1 c}$ наблюдалось у пациентов с исходно более высокими значениями. Компенсация углеводного обмена чаще наблюдалась у пациентов более молодого возраста с меньшей длитель-

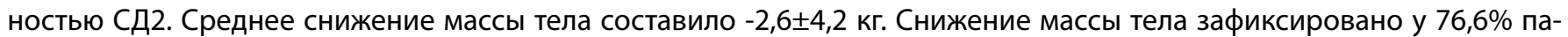
циентов. Наиболее значимое снижение массы тела отмечалось у пациентов с большим исходным индексом массы тела и меньшей длительностью заболевания. Снижение уровня липопротеидов низкой плотности (ЛПНП) отмечено у 74,7\% пациентов. Наиболее выраженное абсолютное снижение ЛПнП наблюдалось у пациентов с исходно более высокими значениями и чаще у лиц более молодого возраста с меньшей длительностью СД2. Среднее снижение

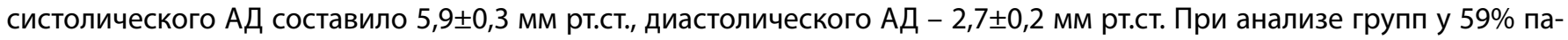
циентов отмечено снижение АД. Наиболее часто снижение АД наблюдалось у пациентов более молодого возраста с меньшей длительностью СД2. При этом более выраженное абсолютное снижение АД наблюдалось у пациентов с исходно более высокими показателями, а повышение, наоборот, - у пациентов с исходно более низкими показателями.

ЗАКЛЮЧЕНИЕ. Интенсификация терапии с помощью алоглиптина у большинства пациентов с СД2 позволяла достичь компенсации углеводного обмена, умеренного снижения массы тела, АД и показателей ЛПнП. Наиболее часто достижение целевых показателей $\mathrm{HbA}_{1 с}$ наблюдалось у пациентов молодого возраста с меньшей длительностью СД2.

КЛЮЧЕВЫЕ СЛОВА: сахарный диабет 2 mипа, ингибиторы ДПП-4, алоглиптин.

\title{
SPECIFIC FEATURES OF THE USE OF ALOGLIPTIN IN VARIOUS GROUPS OF PATIENTS WITH TYPE 2 DIABETES MELLITUS: ADDITIONAL RESULTS OF THE ENTIRE STUDY
}

(c) Marina V. Shestakova ${ }^{*}$, Ekaterina A. Shestakova', Vera A. Kachko²

'Endocrinology Research Centre, Moscow, Russia

${ }^{2}$ Takeda Pharmaceuticals LLC, Moscow, Russia

BACKGROUND: Since the obtaining of data on the effect of Alogliptin towards the lipid profile, body weight and blood pressure (BP) of patients, the additional analysis of the results of the ENTIRE study, completed in the Russian Federation in 2018, was conducted.

AIMS: Assess the dynamics of $\mathrm{HbA}_{1 c^{\prime}}$ body weight, fats indices, blood pressure (BP), and characterize the profile of the patient who received the maximum clinical benefit on treatment of Alogliptin therapy in the ENTIRE study.

MATERIALS AND METHODS: A prospective non-interventional observational study that included patients aged 18 years and older with first-onset type 2 diabetes mellitus (T2DM) or patients with T2DM who did not achieve their glycemic targets during the previous therapy.

RESULTS: A decrease in glycated hemoglobin $\left(\mathrm{HbA}_{1 c}\right)$ by more than $0.5 \%$ was detected in $73.5 \%$ of patients. The most significant absolute decrease of $\mathrm{HbA}_{1 c}$ was noticed in patients with initially higher values. Younger patients with a shorter duration 
of T2DM showed the more often compensation of carbohydrate metabolism. The average loss of weight was $-2.6 \pm 4.2 \mathrm{~kg}$. $76.6 \%$ of patients showed the loss of weight. The most significant decrease in body weight was noticed in patients with a large initial body mass index and a shorter duration of the disease. $74.7 \%$ of patients showed a decrease of the level of low-density lipoproteins (LDL). The most significant absolute decrease in LDL was noticed in patients with initially higher values and more often in younger people with a shorter duration of T2DM. The average decrease in systolic blood pressure (BP) was $5.9 \pm 0.3 \mathrm{~mm} \mathrm{Hg}$; the average decrease in diastolic blood pressure (BP) was $2.7 \pm 0.2 \mathrm{~mm} \mathrm{Hg}$. $59 \%$ of patients showed decrease of blood pressure during the group analyzing. The most frequent BP reduction was observed in younger patients with shorter duration of T2DM. At the same time, a more significant absolute decrease in blood pressure was noticed in patients with initially higher indicators, and an increase, on the contrary, was observed in patients with initially lower indicators.

CONCLUSIONS:The intensification of Alogliptin therapy allowed to achieve the compensation of carbohydrate metabolism, moderate decrease of body weight, blood pressure and LDL indices within the majority of patients with T2DM. The most frequent achievement of $\mathrm{HbA}_{1 \mathrm{c}}$ targets was noticed in young patients with a shorter duration of T2DM.

KEYWORDS: type 2 diabetes mellitus, dipeptidyl peptidase-4 inhibitors, alogliptin.

\section{ОБОСНОВАНИЕ}

Сахарный диабет 2 типа (СД2) представляет собой тяжелое, хроническое и постоянно прогрессирующее заболевание. Характер течения болезни и наличие осложнений в первую очередь обусловлены длительностью заболевания, тяжестью гипергликемии, возрастом пациентов, наличием коморбидной патологии и мотивацией пациентов к лечению. Несмотря на то что в лечении СД2 основную роль играют сами пациенты, тем не менее, сложно недооценивать важность принятия врачебного решения о ранней инициации и/или интенсификации терапии.

По данным российского Федерального регистра сахарного диабета, около половины пациентов на фоне проводимой терапии не достигают целевого уровня $\mathrm{HbA}_{1 c}<7,0 \%$ [1]. Это свидетельствует о том, что проблема своевременной и адекватной интенсификации терапии СД2 в условиях реальной клинической практики требует более гибких подходов с учетом индивидуальных особенностей пациентов.

Согласно алгоритмам персонализации выбора сахароснижающих препаратов (ССП), во многих случаях приоритет отдается современным классам ССП, благоприятно влияющим на сердечно-сосудистый прогноз и снижающим массу тела [1], однако назначения ССП в реальной клинической практике пока расходятся с требованиями рекомендаций.

В РФ наиболее часто назначаемыми классами пероральных ССП являются: в монотерапии - метформин 57,3\%, препараты сульфонилмочевины (СМ) - 41,2\% и ингибиторы дипептидилпептидазы-4 (иДПП-4) - 1\%, все остальные классы составляют менее 1\%; в комбинации 2 ССП - метформин + СМ - 92,6\%, метформин + иДПП-4 - 5,6\% и СМ + иДПП-4 - 0,7\%, все остальные комбинации составляют менее 1\%; в комбинации 3 и более ССП наиболее распространенными вариантами являются метформин + СМ + иДПП-4 (составляет 81,6\%), а также метформин + CM + ингибиторы натрий-глюкозного ко-транспортера 2 (иНГЛТ-2) (около 9\%) [1].

Одним из представителей класса иДПП-4 является алоглиптина бензоат (Випидия $\left.{ }^{\circledR}\right)$, который имеет обширную доказательную базу и был хорошо изучен в ходе нескольких проспективных рандомизированных и пострегистрационных наблюдательных (неинтервенционных) контролируемых исследований как в режиме монотера- пии, так и в комбинации с другими ССП [2-6]. В настоящий момент на сайте clinicaltrials.gov зарегистрировано 58 исследований с применением алоглиптина. В исследовании EXAMINE [6] была отдельно показана высокая кардиоваскулярная безопасность препарата в группе пациентов с СД2, перенесших острый коронарный синдром (15-90-е сутки до рандомизации) - такой выбор самых тяжелых пациентов сильно отличает это исследование от аналогичных исследований других иДПП-4.

В 2016-2018 гг. в РФ было проведено многоцентровое (53 исследовательских центра) проспективное наблюдательное (неинтервенционное) клиническое исследование ENTIRE, направленное на оценку эффективности и безопасности алоглиптина у пациентов с СД2 в условиях реальной клинической практики. Результаты этого исследования были опубликованы в Русском медицинском журнале в 2019 г. [4]. Алоглиптин продемонстрировал значительное улучшение гликемического контроля у исследуемых пациентов при хорошей переносимости и низкой частоте нежелательных явлений [4]. Наряду с высокой эффективностью и безопасностью алоглиптина было показано дополнительное умеренное снижение массы тела, умеренное снижение артериального давления (АД) и уменьшение уровня липидов у пациентов с СД2.

Так, среднее снижение уровня $\mathrm{HbA}_{1 c}$ к концу исследо-

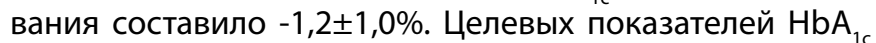
$<7,0 \%$ достигли 52\% пациентов, а уровень снижения $\mathrm{HbA}_{1 с}$ зависел от его исходного уровня. В подгруппе пациентов с наиболее высокими исходными показателями (HbA1c $\geq 9 \%, \mathrm{~N}=248$ (17,8\% от всей выборки)) было достигнуто наиболее выраженное снижение - $-2,5 \pm 1,4 \%$. Показатели $\mathrm{HbA}_{1 c}$ снижались на протяжении всего периода наблюдения [4].

Учитывая полученные данные, касающиеся влияния алоглиптина на уровень липидов и вес пациентов, а также актуальность проблемы рациональной инициации и интенсификации терапии СД2, был проведен дополнительный анализ данных исследования ENTIRE, результаты которого представлены в настоящей публикации.

\section{ЦЕЛЬ}

Цель данного анализа - оценить динамику $\mathrm{HbA}_{1 c^{\prime}}$ массы тела, липопротеидов низкой плотности (ЛПНП), АД и охарактеризовать профиль пациента, получившего 
максимальную клиническую пользу на фоне терапии алоглиптином в ходе исследования ENTIRE.

\section{МЕТОДЫ}

\section{Дизайн исследования}

Исследование ENTIRE представляло собой локальное мультицентровое неинтервенционное проспективное наблюдательное исследование по оценке эффективности алоглиптина в реальной практике лечения пациентов с СД2. Основные результаты и дизайн исследования ENTIRE были описаны ранее [4]. В то же время профили пациентов, которые в большей или меньшей степени ответили на терапию по тем или иным показателям, ранее не описывались и не анализировались, т.к. это не предусматривалось протоколом исследования.

Представленный в настоящей публикации дополнительный анализ данных призван оценить характеристики различных категорий пациентов, в зависимости от уровня снижения $\mathrm{HbA}_{1 c^{\prime}}$ липидов, массы тела и АД.

\section{Критерии соответствия}

В исследование ENTIRE включались пациенты с СД2 в возрасте 18 лет и старше с впервые диагностированным СД2 или пациенты с СД2, не достигшие целевых показателей гликемии. Основными критериями исключения являлись противопоказания к применению алоглиптина, а также применение препаратов группы иДПП-4 или агонистов рецепторов глюкагоноподобного пептида 1-го типа (аГПП-1) в течение 3 месяцев до начала исследования.

\section{Условия проведения}

В исследовании приняли участие 53 исследовательских центра, которые были отобраны в соответствии со следующими критериями: любой тип учреждения системы здравоохранения и высшие профессиональные учреждения системы здравоохранения с наличием амбулаторных отделений, наличие квалифицированных эндокринологов, значительный поток пациентов с СД2 не менее 350 пациентов с СД2 в год.

\section{Продолжительность исследования}

Исследование ENTIRE было проведено в РФ в период с мая 2016 по август 2018 гг. Алоглиптин назначался лечащими врачами в соответствии с показаниями к применению в рамках рутинной клинической практики.

В рамках исследования в течение 6-месячного периода наблюдения было предусмотрено 3 посещения врача (визит 1 - визит включения, визит 2 - через 3 месяца от начала терапии, визит 3 - через 6 месяцев от начала терапии).

\section{Описание медицинского вмешательства}

Все пациенты подписали информированное согласие на визите включения. Отказ от участия в исследовании не влиял на выбор терапии для больных. На каждом визите оценивались уровень $\mathrm{HbA}_{1 c^{\prime}}$ показатели гликемии, липидного спектра, массы тела, АД, нежелательных явлений. На каждого пациента заводилась индивидуальная регистрационная карта, в которой фиксировались все анализируемые параметры.

\section{Исходы исследования}

Проведенный субанализ данных является поисковым по своей сути. Какого-либо предопределенного планирования конечных точек для проведения данного субанализа не осуществлялось и не планировалось изначально. Исходы, первичные и вторичные конечные точки и методы их регистрации в исследовании ENTIRE приведены в первичной публикации.

\section{Методы регистрации исходов}

\section{Анализ в подгруппах}

В рамках настоящего анализа оценивались следующие профили пациентов:

- пациенты с различным уровнем снижения $\mathrm{HbA}_{1 c}$ :

- снижение $\mathrm{HbA}_{1 с}$ более/менее 0,5\%;

- пациенты с различной динамикой массы тела:

- масса тела увеличилась/уменьшилась/без динамики;

- пациенты с различной динамикой показателей липидного спектра:

- повышение/снижение/без динамики уровня липидов;

- пациенты с различной динамикой показателей АД:

- повышение/снижение/без динамики.

\section{Этическая экспертиза}

Исследование было одобрено Независимым междисциплинарным комитетом по этической экспертизе клинических исследований (выписка из протокола №7 от 22.04.2016) и проведено в соответствии с протоколом и этическими принципами Хельсинкской Декларации, а также положениями протоколов Надлежащей практики фармаконадзора, Надлежащей фармакоэпидемиологической практики (НФЭП), руководством по НФЭП Международного общества фармацевтического инжиниринга, требованиями Надлежащей эпидемиологической практики и местных нормативных актов.

\section{Статистический анализ}

Bсе непрерывные переменные были обработаны с использованием показателей описательной статистики. Для всех категориальных переменных использовались частотные и процентные характеристики. Для всех переменных также были рассчитаны точные 95\% доверительные интервалы. Показатель достоверности различий $(p<0,05)$ между группами для всех непрерывных переменных рассчитывался с использованием теста Вилкоксона, для категориальных переменных - с использованием теста Хи-квадрат.

\section{РЕЗУЛЬТАТЫ}

Объекты (участники) исследования

В исследование было включено 1399 участников,

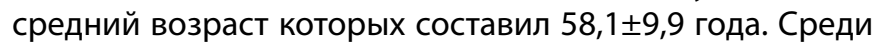
пациентов количество женщин (64,8\%) преобладало над количеством мужчин. Основные демографические и анамнестические данные пациентов представлены в таблице 1. Согласно протоколу исследования, не требовалось документального подтверждения наличия сопутствующих заболеваний. 
Таблица 1.Исходные характеристики исследуемой популяции

\begin{tabular}{|c|c|c|}
\hline & Характеристика & $\begin{array}{c}\text { Bсего } \\
\text { N (\%) }[95 \% \text { ДИ] }\end{array}$ \\
\hline \multirow{5}{*}{ Возрастная группа } & Возраст & $58,1 \pm 9,9$ \\
\hline & $<52$ лет & $341(24,4 \%)[21,3-27,7]$ \\
\hline & 52-59 лет & $331(23,7 \%)[20,6-27,0]$ \\
\hline & 59-65 лет & $356(25,4 \%)[22,3-28,8]$ \\
\hline & $\geq 65$ лет & $371(26,5 \%)[23,4-29,9]$ \\
\hline \multirow{2}{*}{ Пол } & Женщины & $907(64,8 \%)[62,3-67,3]$ \\
\hline & Мужчины & $492(35,2 \%)[32,7-37,7]$ \\
\hline \multirow{3}{*}{ Курение } & Никогда не курили & $1099(78,6 \%)[75,8-81,1]$ \\
\hline & Ранее курили & $190(13,6 \%)[11,5-16,0]$ \\
\hline & Курят & $110(7,9 \%)[6,3-9,8]$ \\
\hline \multirow{5}{*}{ ИМТ } & Нормальная масса тела (ИМТ<25 кг/м²) & $78(5,6 \%)[4-7,8]$ \\
\hline & 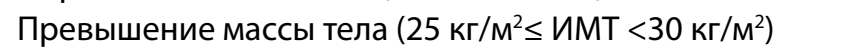 & $396(28,4 \%)[24,8-32,2]$ \\
\hline & 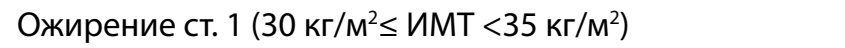 & $530(38 \%)[34,1-42,1]$ \\
\hline & 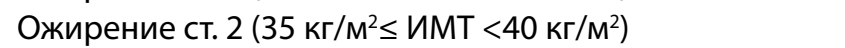 & $275(19,7 \%)[16,6-23,2]$ \\
\hline & Ожирение ст. 3 (ИМТ $\geq 40$ кг/м²) & $116(8,3 \%)[6,3-10,9]$ \\
\hline \multirow{10}{*}{$\begin{array}{l}\text { История сердечно-сосудистых } \\
\text { заболеваний }\end{array}$} & Артериальная гипертония & 999 (71,4\%) [68,4-74,3] \\
\hline & Хроническая сердечная недостаточность & $213(15,2 \%)[13,0-17,7]$ \\
\hline & Аритмия & $68(4,9 \%)[3,6-6,5]$ \\
\hline & Заболевания периферических артерий & $92(6,6 \%)[5,1-8,4]$ \\
\hline & Коронарная болезнь сердца & $257(18,4 \%)[16,0-21,0]$ \\
\hline & Инфаркт миокарда & $76(5,4 \%)[4,1-7,1]$ \\
\hline & Инсульт & $42(3 \%)[2,1-4,3]$ \\
\hline & Дислипидемия & $848(60,6 \%)[57,4-63,8]$ \\
\hline & Другие сердечно-сосудистые заболевания & $27(1,9 \%)[1,2-3,1]$ \\
\hline & Семейная история сердечно-сосудистых заболеваний & $532(38 \%)[34,9-41,2]$ \\
\hline \multirow{5}{*}{ Продолжительность СД2 } & Средняя продолжительность & $4,5 \pm 5,1$ \\
\hline & 0-3 года & $738(52,8 \%)[49,1-56,5]$ \\
\hline & 3-5 лет & $145(10,4 \%)[8,3-12,9]$ \\
\hline & 5-10 лет & $314(22,5 \%)[19,5-25,7]$ \\
\hline & $\geq 10$ лет & $200(14,3 \%)[11,9-17,1]$ \\
\hline \multirow{4}{*}{ История СД2 и его осложнений } & Диабетическая ретинопатия & $186(13,3 \%)[11,6-15,2]$ \\
\hline & Автономная нейропатия & $317(22,7 \%)[20,5-24,9]$ \\
\hline & Диабетическая нефропатия & $93(6,6 \%)[5,4-8,1]$ \\
\hline & Семейная история СД2 & $550(39,3 \%)[36,2-42,5]$ \\
\hline \multirow{7}{*}{$\begin{array}{l}\text { Сахароснижающая терапия до } \\
\text { включения в исследование }\end{array}$} & Не получали сахароснижающую терапию & $353(25,2 \%)[21,3-29,6]$ \\
\hline & Монотерапия бигуанидами & $661(47,2 \%)[42,6-52,0]$ \\
\hline & Монотерапия препаратами сульфонилмочевины & $85(6,1 \%)[4,2-8,8]$ \\
\hline & Другая монотерапия & $1(0,1 \%)[0,0-1,0]$ \\
\hline & Бигуаниды + препараты сульфонилмочевины & $281(20,1 \%)[16,6-24,1]$ \\
\hline & Бигуаниды + ингибиторы НГЗТ-2 & $11(0,8 \%)[0,3-2,2]$ \\
\hline & Другая комбинированная терапия & $7(0,5 \%)[0,1-1,7]$ \\
\hline \multirow{4}{*}{ Сердечно-сосудистый риск* } & Очень высокий & $1216(86,9 \%)[85-88,6]$ \\
\hline & Высокий & $183(13,1 \%)[11,4-15]$ \\
\hline & Макроваскулярные осложнения & $1098(78,5 \%)[75,7-81]$ \\
\hline & Микроваскулярные осложнения & $954(68,2 \%)[65,7-70,6]$ \\
\hline \multirow{6}{*}{ Лабораторные показатели } & Средний $\mathrm{HbA}_{1 \mathrm{c}}(\%)$ & $8,2 \pm 1,2$ \\
\hline & $7,5 \% \leq \mathrm{HbA}_{1 c}<9 \%$ & $749(53,8 \%)[50,5-57,1]$ \\
\hline & $\mathrm{HbA}_{1 \mathrm{c}}<-7,5 \%$ & $395(28,4 \%)[25,5-31,4]$ \\
\hline & $\mathrm{HbA}_{1 \mathrm{c}} \geq 9 \%$ & $248(17,8 \%)[15,4-20,5]$ \\
\hline & Уровень глюкозы натощак, ммоль/л & $8,7 \pm 2,1$ \\
\hline & Постпрандиальный уровень глюкозы, ммоль/л & $10,4 \pm 2,2$ \\
\hline \multirow{2}{*}{$\begin{array}{l}\text { Назначенная дозировка } \\
\text { препарата }\end{array}$} & $25 \mathrm{Mr}$ & $1349(96,4 \%)[95-97,5]$ \\
\hline & $12,5 \mathrm{Mr}$ & $50(3,6 \%)[2,5-5]$ \\
\hline
\end{tabular}

* - К очень высокому риску были отнесены пациенты с любым из указанных симптомов: артериальная гипертензия, дислипидемия, семейная история сердечно-сосудистых заболеваний, диабетическая ретинопатия, диабетическая нефропатия, автономная нейропатия в истории или текущий статус курильщика. Остальным пациентам присваивался статус высокого риска. Для средних данных после \pm приведено стандартное отклонение. 
Основные результаты исследования

В соответствии с целью дополнительного анализа исследования ENTIRE были проанализированы группы пациентов, в которых была отмечена наиболее выраженная динамика $\mathrm{HbA}_{1 c^{\prime}}$ массы тела, показателей липидного спектра и артериального давления.

При оценке липидного профиля к завершению исследования средний уровень общего холестерина умень-

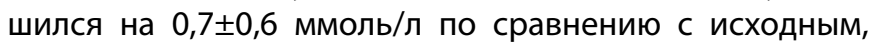
и изменение данного показателя оказалось статистически и клинически значимым для группы пациентов с исходным значением $\mathrm{HbA}_{1 c} \geq 7,5 \%$, но <9,0\% (p<0,001). Среднее снижение показателей триглицеридов составило 0,5 $\pm 0,5$ ммоль/л $(p<0,001)$, уровня ЛПНП $0,6 \pm 0,6$ ммоль/л $(p<0,001)$ от исходного значения, уровень ЛПВП значимо не изменился. Существенных различий по этим показателям между исследуемыми подгруппами выявлено не было [4].

Также было продемонстрировано значимое $(p<0,001)$ снижение массы тела исследуемых пациентов на фоне применения алоглиптина [4]. Среднее снижение массы

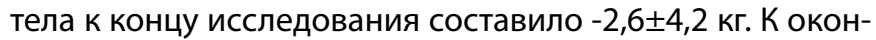
чанию исследования изменение массы тела было наиболее значимым в группе пациентов с исходным $\mathrm{HbA}_{1 с}$ $<7,5 \%--3 \pm 4,6$ кг, тогда как в группе пациентов с $\mathrm{HbA}_{1 c}$ 7,5-9,0\% этот показатель составил $-2,6 \pm 4$ кг, а в группе пациентов с $\mathrm{HbA}_{1 \mathrm{c}} \geq 9,0 \%-2,3 \pm 4,4$ кг.

Средние показатели динамики АД, липидного спектра, массы тела представлены на рисунке 1.

Профили пациентов со снижением $\mathrm{HbA}_{1 с}$ более $-0,5 \%$ и менее $-0,5 \%$

В рамках основной публикации было показано, что наибольшие показатели снижения $\mathrm{HbA}_{1 c}$ отмечаются у пациентов с более высокими исходными показателями $\mathrm{HbA}_{1 c}$. Данные результаты были подтверждены в том числе в модели множественной логистической регрессии. Пациенты с $\mathrm{HbA}_{1 c}>9 \%$ показали снижение исследуемого показателя в среднем на $-2,5 \pm 1,4 \%$, пациенты с $\mathrm{HbA}_{1 c}$ в диапазоне 7,5-9,0 - на $-1,1 \pm 0,6 \%$, а пациенты с $\mathrm{HbA}_{1 c}$ $<7,5-$ на $-0,6 \pm 0,5 \%$.
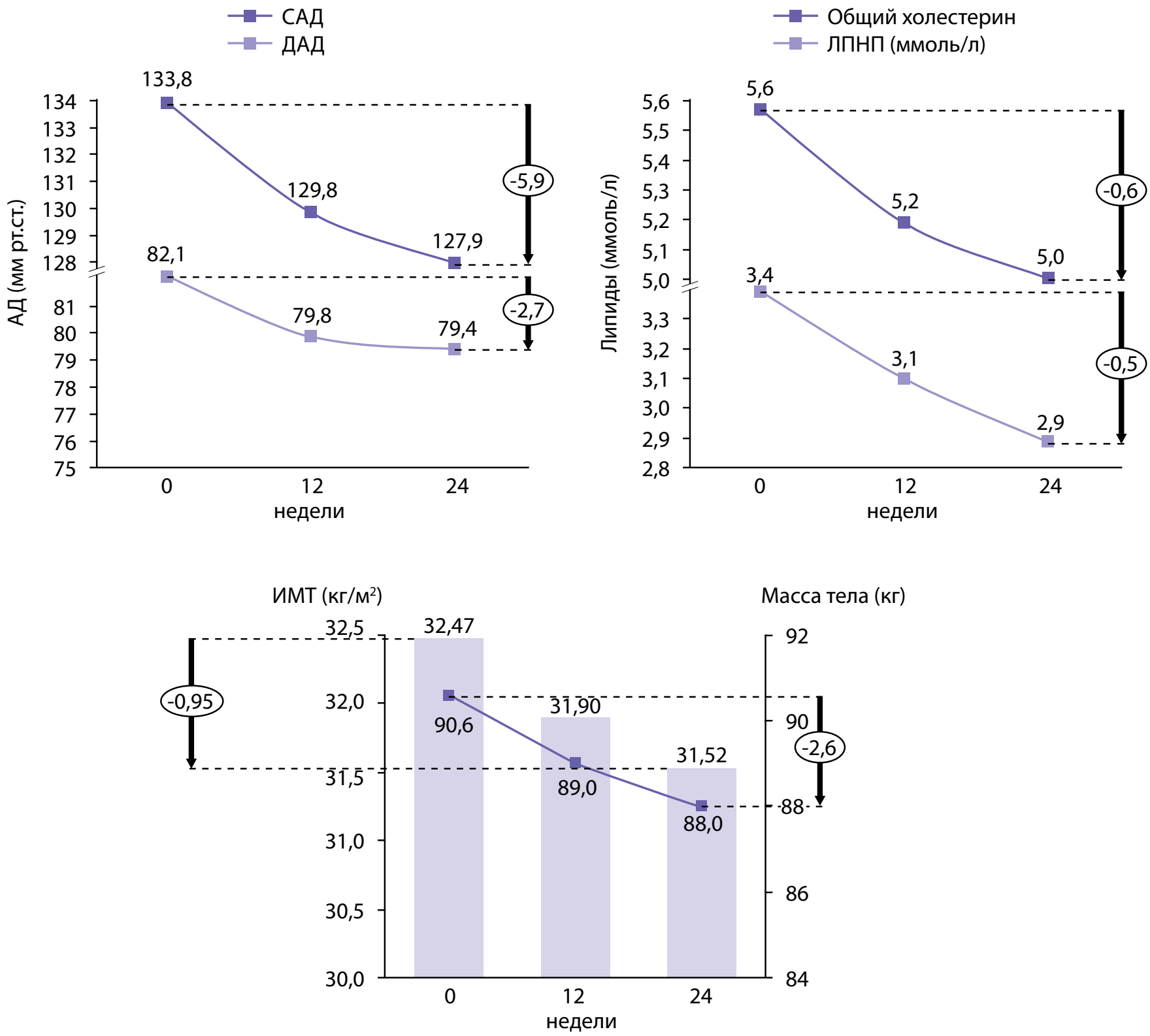

Рисунок 1. Результаты исследования ENTIRE: внепанкреатические эффекты терапии (данные представлены в виде средних значений) 
Большая часть пациентов была представлена в категории снизивших $\mathrm{HbA}_{1 с}$ более чем на 0,5\%: 1106 пациентов, или 73,5\%, и у 293 пациентов (26,5\%) HbА снизился менее чем на 0,5\%.

Пациенты, в большей степени ответившие на терапию, характеризовались более высоким уровнем $\mathrm{HbA}_{1 c}$ на исходном визите. В группе пациентов со снижением $\mathrm{HbA}_{1 с}$ менее -0,5\% исходный $\mathrm{HbA}_{1 с}$ составил 7,42\%, 95\% ДИ [7,33-7,51], и в группе с динамикой $\mathrm{HbA}_{1 с}$ более $-0,5 \%$ исходный $\mathrm{HbA}_{1 с}$ был 8,34\%, 95\% ДИ [8,27-8,41], p<0,0001. Аналогичным образом данные подгруппы пациентов значимо различались и по другим показателям углеводного обмена, а также по длительности анамнеза СД2 соответственно: 5,16 года, 95\% ДИ [4,6-5,72] и 4,35 года, 95\% ДИ [4,04-4,65], p<0,0001. При этом достоверные отличия были получены и в отношении возраста пациентов на момент включения в исследование: 59,31 года, 95\% ДИ [58,18-60,43] и 57,74 года, 95\% ДИ [57,15-58,32] соответственно, $\mathrm{p}<0,007$.

Таким образом, пациенты более молодого возраста (57,7 года), с меньшей длительностью СД2 (4,3 года) и с более высокими исходными показателями углеводного обмена показывают более выраженное снижение $\mathrm{HbA}_{1 c^{\prime}}$ постпрандиальной гликемии (ППГ), глюкозы плазмы натощак (ГПН) (табл. 2). Статистически значимых отличий по липидному профилю, ЧСС и АД получено не было.

Профили пациентов с различной динамикой мас-

\section{сы тела}

Снижение массы тела зафиксировано в большей части случаев у 76,6\% пациентов, включенных в исследование $(\mathrm{n}=1068)$. Не было отмечено динамики массы тела в $10,7 \%$ случаев $(n=150)$, масса тела повысилась у $12,9 \%$ пациентов $(n=181)$. В связи с неинтервенционным, наблюдательным характером исследования для анализа данных было введено предположение, что у пациентов без изменений массы тела результаты обусловлены не только отсутствием динамики этого показателя за время наблюдения, но и, возможно, отсутствием повторных измерений, в связи с чем показатель достоверности рассчитывался только для пары «повышение» и «снижение».

Результаты данного попарного сравнения показали, что в группе снижения массы тела отмечалось более выраженное снижение $\mathrm{HbA}_{1 c}-1,24$ [-1,3--1,18] в сравнении c-1,03 [-1,2--0,85] в группе повышения массы тела, $p<0,001$. Аналогичные изменения были зафиксированы и для других показателей углеводного обмена, ГПН, ППГ. При этом исходный $\mathrm{HbA}_{1 с}$ в подгруппе пациентов, снизивших вес, был достоверно ниже: 8,09\% в подгруппе пациентов, снизивших вес, и 8,37\% в группе, у которой было отмечено повышение массы тела, $p<0,05$. Таким образом, у пациентов из группы снижения массы тела наблюдался больший ответ на проводимую сахароснижающую терапию. Сравнительная характеристика пациентов в группах снижения и увеличения массы тела представлена в таблице 3.

Следует отметить, что большее снижение массы тела наблюдалось у более молодых пациентов с меньшей продолжительностью СД2, которые лучше отвечали на проводимую сахароснижающую терапию, что выражалось в большем снижении показателей $\mathrm{HbA}_{1 c^{\prime}}$ ГПН и ППГ.

\section{Профили пациентов с различной динамикой} ЛПНП

У исследуемых пациентов среди всех показателей липидного обмена наиболее выраженную динамику выявили в уровне ЛПНП. Если среднее снижение общего холестерина на фоне применения алоглиптина составило 10,7\%, то снижение уровня ЛПНП - 17,6\%.

В целом показатель ЛПНП, в отличие от массы тела или показателей углеводного обмена, оценивался в исследовании реже, что объяснимо другими основными целями исследования и неинтервенционным характером исследования. Общее количество пациентов, у которых оценивались показатели ЛПнП, было меньше

Таблица 2. Профили пациентов со снижением гликированного гемоглобина более или равно и менее -0,5\%

\begin{tabular}{|c|c|c|c|}
\hline Показатель & $\begin{array}{c}\text { Снижение менее или } \\
\text { равно -0,5 }\end{array}$ & Снижение более -0,5 & $\mathbf{p}$ \\
\hline $\begin{array}{l}\text { Снижение } \mathrm{HbA}_{1 с} \text { от } \\
\text { исходного, ммоль/л }\end{array}$ & $\begin{array}{c}-0,22 \\
{[-0,26--0,17]}\end{array}$ & $\begin{array}{c}-1,48 \\
{[-1,54--1,42]}\end{array}$ & $<0,001$ \\
\hline Возраст, лет & $\begin{array}{c}59,31 \\
{[58,18-60,43]}\end{array}$ & $\begin{array}{c}57,74 \\
{[57,15-58,32]}\end{array}$ & 0,007 \\
\hline $\mathrm{HbA}_{1 с}$ исходно, \% & $\begin{array}{c}7,42 \\
{[7,33-7,51]}\end{array}$ & $\begin{array}{c}8,34 \\
{[8,27-8,41]}\end{array}$ & $<0,001$ \\
\hline $\mathrm{HbA}_{1 с}$ на визите $3, \%$ & $\begin{array}{c}7,2 \\
{[7,1-7,3]}\end{array}$ & $\begin{array}{c}6,91 \\
{[6,87-6,96]}\end{array}$ & $<0,001$ \\
\hline ГПН исходно, ммоль/л & $\begin{array}{c}8,26 \\
{[8,05-8,47]}\end{array}$ & $\begin{array}{c}8,84 \\
{[8,71-8,98]}\end{array}$ & $<0,001$ \\
\hline ППГ исходно, ммоль/л & $\begin{array}{c}9,73 \\
{[9,43-10,02]}\end{array}$ & $\begin{array}{c}10,56 \\
{[10,4-10,71]}\end{array}$ & $<0,001$ \\
\hline Масса тела исходно, кг & $\begin{array}{c}89,74 \\
{[87,84-91,63]}\end{array}$ & $\begin{array}{c}90,79 \\
{[89,82-91,76]}\end{array}$ & 0,314 \\
\hline ИМТ исходно, кг/м² & $\begin{array}{c}32,7 \\
{[32,1-33,31]}\end{array}$ & $\begin{array}{c}32,41 \\
{[32,09-32,73]}\end{array}$ & 0,256 \\
\hline $\begin{array}{l}\text { Длительность течения СД2, } \\
\text { лет }\end{array}$ & $\begin{array}{c}5,16 \\
{[4,6-5,72]}\end{array}$ & $\begin{array}{c}4,35 \\
{[4,04-4,65]}\end{array}$ & $<0,001$ \\
\hline
\end{tabular}


Снижение ( $n=1068)$

\begin{tabular}{|c|c|}
\hline $\begin{array}{l}\text { Снижение } \mathrm{HbA}_{1 \mathrm{c}} \text { от } \\
\text { исходного, ммоль/л }\end{array}$ & $\begin{array}{c}-1,24 \\
{[-1,3--1,18]}\end{array}$ \\
\hline Возраст, лет & $\begin{array}{c}57,52 \\
{[56,91-58,12]}\end{array}$ \\
\hline $\mathrm{HbA}_{1 с}$ исходно, \% & $\begin{array}{c}8,09 \\
{[8,02-8,16]}\end{array}$ \\
\hline $\mathrm{HbA}_{1 \mathrm{c}}$ на визите 3, \% & $\begin{array}{c}6,87 \\
{[6,83-6,92]}\end{array}$ \\
\hline ГПН исходно, ммоль/л & $\begin{array}{c}8,71 \\
{[8,58-8,84]}\end{array}$ \\
\hline ППГ исходно, ммоль/л & $\begin{array}{c}10,25 \\
{[10,1-10,4]}\end{array}$ \\
\hline Масса тела исходно, кг & $\begin{array}{c}92,77 \\
{[91,81-93,72]}\end{array}$ \\
\hline ИМТ исходно, кг/м² & $\begin{array}{c}33,03 \\
{[32,72-33,34]}\end{array}$ \\
\hline ГПН на визите 3, ммоль/л & $\begin{array}{c}6,47 \\
{[6,41-6,53]}\end{array}$ \\
\hline ППГ на визите 3, ммоль/л & $\begin{array}{c}7,84 \\
{[7,77-7,91]}\end{array}$ \\
\hline Масса тела на визите 3, кг & $\begin{array}{c}89,03 \\
{[88,1-89,96]}\end{array}$ \\
\hline ИМТ на визите $3, \mathrm{kг} / \mathrm{M}^{2}$ & $\begin{array}{c}31,66 \\
{[31,35-31,97]}\end{array}$ \\
\hline Динамика массы тела, кг & $\begin{array}{c}-3,86 \\
{[-4,1--3,61]}\end{array}$ \\
\hline Динамика ИМТ, кг/м² & $\begin{array}{c}-1,36 \\
{[-1,45--1,28]}\end{array}$ \\
\hline $\begin{array}{l}\text { Длительность течения СД2, } \\
\text { лет }\end{array}$ & $\begin{array}{c}4,1 \\
{[3,81-4,39]}\end{array}$ \\
\hline
\end{tabular}

Повышение $(n=181)$

\section{p (для пары «Снижение-повышение»}


77,59 мм рт.ст.; 95\% ДИ [76,55-78,62] и 84,41 мм рт.ст.; 95\% ДИ [83,85-84,97]; $p<0,0001$.

Важно отметить, что в группе, показавшей снижение АД, было также отмечено более выраженное снижение показателей массы тела и ИМТ. Для динамики массы тела эти показатели составили: -3,02 кг; 95\% ДИ [-3,33--2,71] в группе снижения АД в сравнении с -1,99 кг; 95\% ДИ [-2,53--1,45] в группе пациентов, у которых среднее АД повысилось за время исследования, $\mathrm{p}<0,0001$. Для ИМТ:

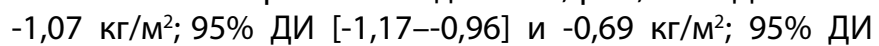
[-0,88--0,5] соответственно, $p<0,0001$. Различная динамика данных показателей отмечалась на фоне исходно не различавшихся массы тела и, соответственно, ИМТ.

Группа снижения АД имела меньшую длительность анамнеза СД2: 4,52 года; 95\% ДИ [4,16-4,87] в сравнении с 5,41 года; 95\% ДИ [4,75-6,06], в группе с повышением АД, $\mathrm{p}<0,023$.

При анализе биохимических показателей не было выявлено различий в показателях углеводного обмена,
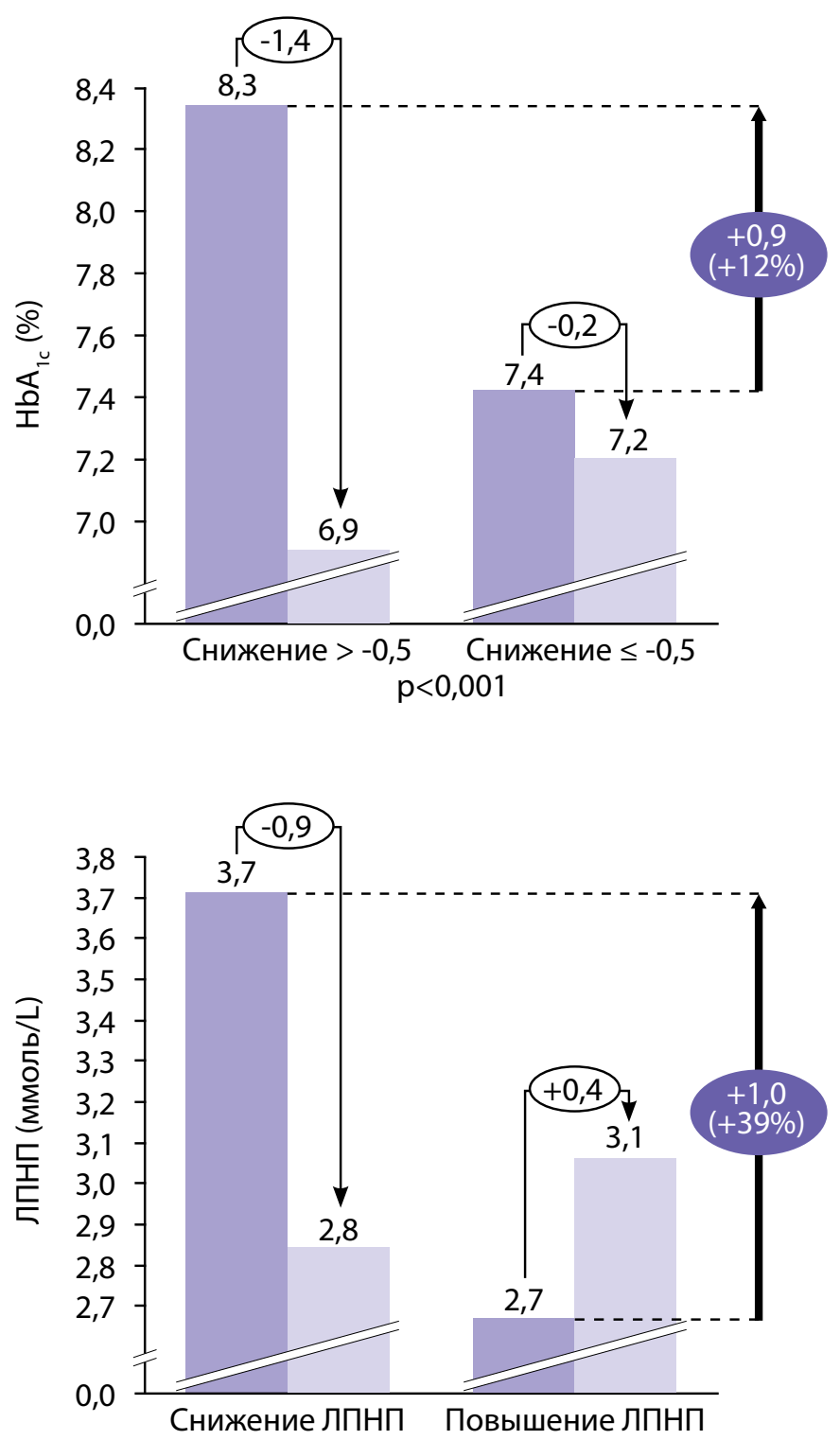

- Исходно за исключением более высоких исходных показателей общего холестерина, триглицеридов и ЛПНП.

Средние значения динамики $\mathrm{HbA}_{1 c^{\prime}}$ АД, массы тела, ЛПНП в подгруппе пациентов с максимальным/минимальным изменением данного показателя представлены на рисунке 2.

\section{ОБСУЖДЕНИЕ}

Снижение $\mathrm{HbA}_{1 c}$

В качестве критерия оценки клинической эффективности терапии в рамках данного субанализа было выбрано снижение $\mathrm{HbA}_{1 c}$ на 0,5\% и более. Результаты проведенного анализа показали, что пациенты более молодого возраста, с меньшей длительностью СД2 и с более высоким исходным значением $\mathrm{HbA}_{1 с}$ показывают более выраженную динамику нормализации $\mathrm{HbA}_{1 c^{\prime}}$ ГПН и ППГ. При этом статистически значимых отличий по липидному профилю, ЧСС и АД получено не было.
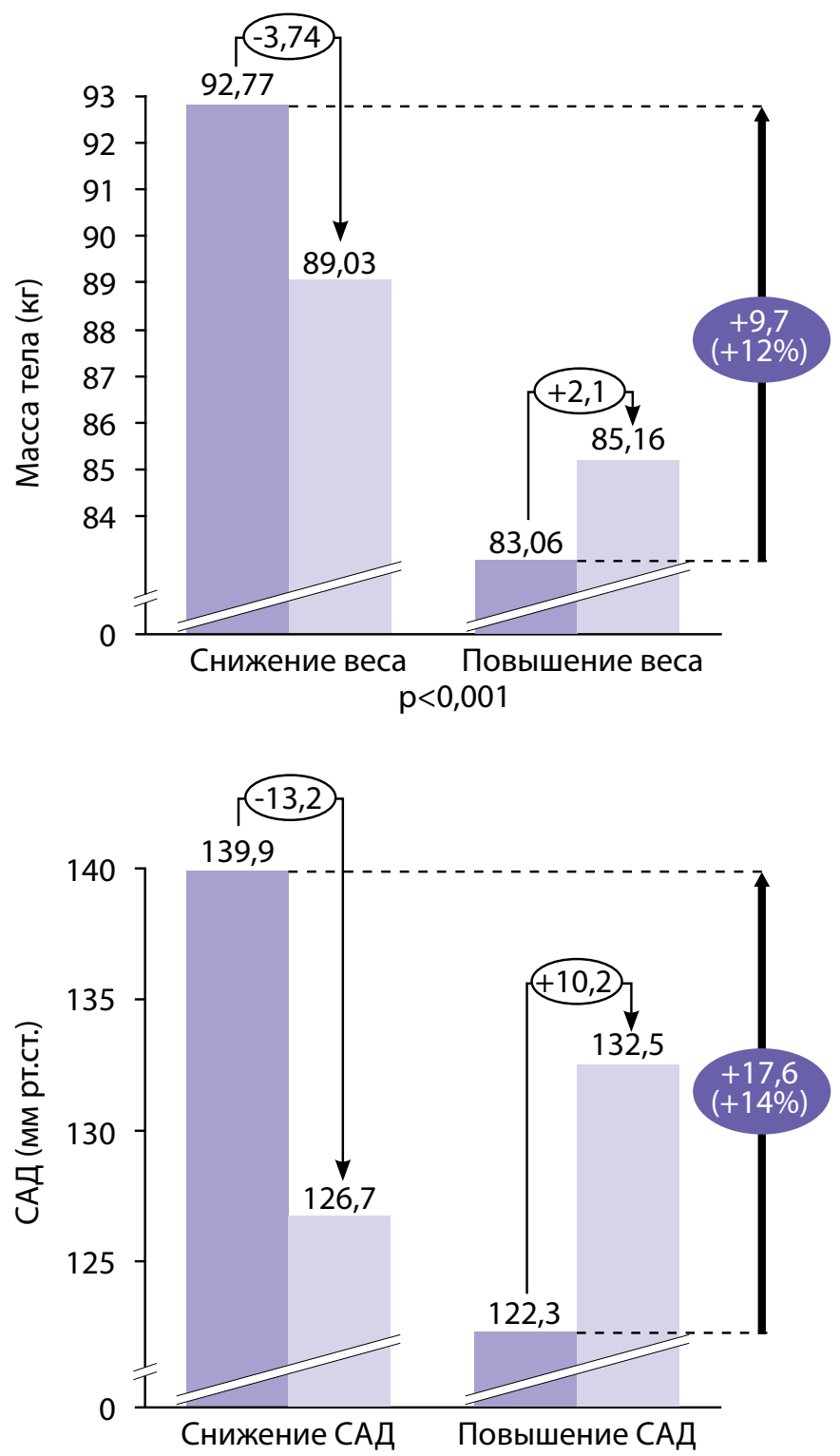

Финально 
Данные результаты соответствуют ранее опубликованным предикторам снижения $\mathrm{HbA}_{1 c}$ [7], которые были выполнены в модели пациентов, получающих препараты инсулина, где было показано, что повышение $\mathrm{HbA}_{1 с}$ в исходной популяции пациентов на 1,0\% приводит к дополнительному снижению финального $\mathrm{HbA}_{1 c}$ на 0,7-0,8\% [7]. В рамках данного анализа исходные средние показатели $\mathrm{HbA}_{1 с}$ для группы со снижением $\leq 0,5 \%$ составили 7,42\% и для группы со снижением >0,5\% - 8,34\%.

Важно отметить, что абсолютное снижение $\mathrm{HbA}_{1 c}$ было наиболее выраженным у пациентов с исходным более высоким значением $\mathrm{HbA}_{1 c^{\prime}}$ при этом частота достижения цели $\mathrm{HbA}_{1 c}<7 \%$ была выше у более молодых пациентов с меньшим исходным значением $\mathrm{HbA}_{1 с}$ и меньшей длительностью СД2 [7]. Полученные результаты свидетельствуют о том, что назначение алоглиптина является наиболее рациональным у пациентов на ранних стадиях СД2, так как именно такие пациенты с наибольшей вероятностью достигают целевых значений $\mathrm{HbA}_{1 с}$.

\section{Снижение массы тела}

Увеличение массы тела увеличивает риск развития СД2 и осложнений, связанных с диабетом. Более $80 \%$ пациентов с СД2 имеют лишнюю массу тела или ожирение. Люди с избыточным весом более склонны к развитию СД2, и снижение массы тела улучшает прогноз пациентов с СД2. Кроме того, ИМТ является мощным и модифицируемым фактором риска при СД2.

Ингибиторы ДПП-4 в соответствии с данными метаанализов - это класс препаратов с нейтральным влиянием на массу тела. Однако в рамках некоторых исследований при добавлении к терапии иДПП-4 было показано снижение массы тела.

В исследовании ENDURE [8], целью которого была оценка эффективности и безопасности терапии алоглиптином по сравнению с терапией глипизидом в комбинации с метформином, было получено среднее снижение массы тела -0,68 и -0,89 для группы алоглиптина 12,5 и 25 мг соответственно и повышение массы тела на 0,95 кг для группы пациентов, получавших глипизид ( $<<0,001$ для обеих дозировок в сравнении с глипизидом).

Результаты наблюдательной программы «Диа-Да» по изучению эффективности и безопасности применения препарата из группы ингибиторов ДПП-4 ситаглиптина в комбинации с метформином в регионах Российской Федерации показали, что достижение целевых значений гликемии не сопровождалось увеличением массы тела. В то же время через 6 мес терапии имелась отчетливая, но недостоверная тенденция к снижению массы тела [9].

В рандомизированном исследовании VERIFY [10] оценивались результаты использования ингибитора ДПП-4 вилдаглиптина в терапии пациентов в дебюте СД2. За время 5-летнего наблюдения в ходе VERIFY, кроме более длительного удержания целевых значений $\mathrm{HbA}_{1 c}$ на фоне ранней комбинированной терапии, было показано снижение

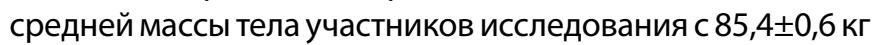
до 84,5 0 ,6 кг. При этом динамика кривых изменения массы тела в VERIFY [10] демонстрирует наиболее выраженное снижение в самом начале лечения, в течение первых 3-6 мес наблюдения за пациентами.
Следует отметить, что в исследовании ENTIRE снижение массы тела также было отмечено в большей степени в популяции пациентов с меньшей длительностью СД2.

\section{Динамика Лпнп}

В 2012 г. Monami и соавт. был выполнен метаанализ результатов рандомизированных клинических исследований [11], в котором, среди прочих, оценивались экстрагликемические эффекты ингибиторов ДПП-4. В этой работе, так же как и в ENTIRE, было показано снижение показателей общего холестерина и триглицеридов (среднее снижение общего холестерина составило $-0,18,95 \%$ ДИ [-0,29--0,06] ммоль/л, p=0,002). Клиническое значение этого небольшого, но статистически значимого различия является спорным, но тем не менее, данный эффект упоминается как возможный благоприятный «непрямой» эффект ингибиторов ДПП-4.

Снижение ЛПНП в ENTIRE было более выраженным: -0,6 ммоль/л от исходного значения к визиту 3 в общей популяции пациентов. При выделении группы пациентов, которые снизили этот показатель в данном субанализе данных, результат составил -0,87, 95\% ДИ [-0,94-0,79] ммоль/л. Возможным объяснением большего снижения ЛПНП в ENTIRE, чем в метаанализе Monami, может быть большая гетерогенность данных, которые использовались при создании метаанализа, а также то, что российская популяция пациентов ENTIRE исходно включала пациентов с относительно более высокими показателями общего холестерина, ЛПНП и триглицеридов, а также с большими ИМТ и массой тела соответственно.

Следует отметить, что оценка влияния алоглиптина на прогрессирование атеросклероза уже выполнялась ранее в рамках отдельного проспективного многоцентрового сравнительного рандомизированного исследования SPEAD-A [12]. Данное исследование было посвящено оценке динамики толщины комплекса интима-медиа у пациентов с СД2. В данной работе в течение 2 лет наблюдения была показана существенная клинически значимая положительная динамика данного показателя на фоне применения алоглиптина по сравнению со стандартной терапией (динамика уменьшения толщины комплекса интима-медиа, определенная с использованием ультразвуковой допплерографии (УЗДГ) брахиоцефальных артерий, составила -0,026 мм, в сравнении с +0,005 мм, P=0,013). Кроме снижения этого показателя в группе пациентов, леченных алоглиптином, было также показано снижение ЛПНП на -0,4 ммоль/л. Ряд исследователей объясняют полученные результаты изменением регуляции липопротеинового транспорта в кишечнике и, как следствие, снижением объема циркулирующих хиломикронов [13, 14].

\section{Артериальное давление}

Препараты группы иДПП-4 используются в качестве гипогликемических препаратов. Тем не менее в дополнение к сахароснижающему эффекту неоднократно было показано влияние на показатели АД. Ранее проведенные исследования показали, что влияние иДПП-4 на АД происходит через сложный механизм, связанный с нервной регуляцией, влиянием на функцию почек, тонус кровеносных сосудов [15-20]. Есть предположения, 
Таблица 4. Клинические характеристики, ассоциированные с выраженным эффектом снижения показателей углеводного обмена, липидов, артериального давления и массы тела

\begin{tabular}{lccc}
\hline $\begin{array}{c}\text { Более выраженное } \\
\text { снижение }\end{array}$ & Более молодой возраст & $\begin{array}{c}\text { Более высокие исходные } \\
\text { показатели }\end{array}$ & $\begin{array}{c}\text { Небольшая длительность } \\
\text { СД 2 типа }\end{array}$ \\
\hline $\mathrm{HbA}_{1 с}$ & Да & Да & Да \\
Липиды & Нет & Да & Нет \\
Масса тела & Да & Да & Да \\
АД & Нет & Да & Да \\
\hline
\end{tabular}

что иДПП-4 улучшают эндотелиальную функцию сосудов у пациентов с гипертонической болезнью, подавляя воспалительные реакции и снижая интенсивность реакций окислительного стресса [21]. Анализ данных более чем 2000 пациентов, ранее не получавших лечения по поводу СД2, которым был назначен иДПП-4 [17], показал снижение систолического АД (с исходных 132,5 $\pm 0,32$

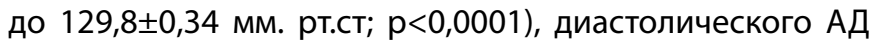

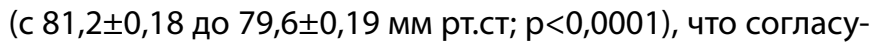
ется с результатами исследования ENTIRE. При этом, как и в ENTIRE, было продемонстрировано снижение уров-

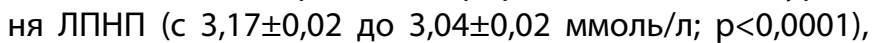

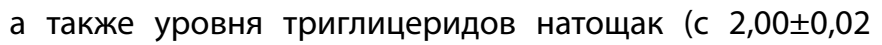

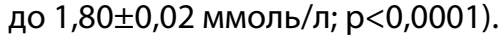

\section{Заключение по дискуссии}

На основании данных исследования ENTIRE в условиях реальной клинической практики назначение алоглиптина пациентам в дебюте заболевания, а также добавление препарата к текущей сахароснижающей терапии позволяло достичь целевых значений показателей углеводного обмена, а также получить ряд дополнительных преимуществ, таких как снижение массы тела, уменьшение выраженности дислипидемии и снижение АД у большинства пациентов.

Полученные данные говорят о том, что отвечают на терапию алоглиптином более выраженным снижением $\mathrm{HbA}_{1 c}$ пациенты более молодого возраста с небольшой длительностью СД2. При этом в абсолютных значениях гликированный гемоглобин, ЛПНП и АД снижались в большей степени у тех пациентов, которые имели более высокие исходные значения данных параметров (табл. 4).

Полученные результаты свидетельствуют о важности корректного сбора и интерпретации клинико-анамнестических и демографических данных при подборе ССП в условиях рутинной клинической практики. В свою очередь, адекватная гипогликемическая терапия является залогом повышения качества и продолжительности жизни пациентов с СД2.

\section{Ограничения исследования}

Как и любое неинтервенционное исследование, данная работа имеет свои ограничения, связанные с отсутствием рандомизации и группы контроля.

На полученные результаты могли повлиять дополнительные назначения препаратов, оказывающих воздействие на показатели липидов, АД, и др., которые, в соответствии с наблюдательным характером исследования, могли осуществляться лечащими врачами. К сожалению, характер имеющейся базы данных не позволяет вычленить пациентов без дополнительных назначений.

Тем не менее значительный объем выборки пациентов позволяет сделать вывод о том, каким пациентам в реальной практике назначается препарат алоглиптин и каких клинических эффектов можно ожидать от данного решения.

Также необходимо понимать, что данная работа представляет собой субанализ данных исследования, которое не было изначально запланировано для того, чтобы оценить влияние тех факторов, о которых шла речь в данной работе.

Кроме того, при оценке данной работы следует принимать во внимание и критерии включения и исключения из исследования, которые могут ограничивать интерпретацию данной работы для клинической практики. Тем не менее полученные данные позволяют рассматривать результаты в качестве поисковых гипотез для дальнейших исследований в этой области.

\section{ЗАКЛЮЧЕНИЕ}

Назначение алоглиптина в качестве монотерапии или в добавление к текущей сахароснижающей терапии было оценено в рутинной клинической практике на российской популяции в исследовании ENTIRE. Интенсификация терапии с помощью алоглиптина у большинства пациентов позволяла достичь компенсации углеводного обмена и умеренного снижения массы тела, АД и показателей ЛПНП. Наиболее часто достижение целевых показателей $\mathrm{HbA}_{1 c}$ наблюдалось у пациентов молодого возраста с меньшей длительностью СД2.

\section{ДОПОЛНИТЕЛЬНАЯ ИНФОРМАЦИЯ}

Источник финансирования. Исследование проведено при финансовом обеспечении ООО «Такеда Фармасьютикалс».

Конфликт интересов. Шестакова М.В. как национальный координатор исследования принимала участие в экспертных советах, связанных с проведением исследования.

Качко В.А. - медицинский советник компании ООО «Такеда Фармасьютикалс».

Участие авторов. Шестакова М.В., Шестакова Е.А. и Качко В.А. принимали участие в анализе полученных данных и написании текста, все авторы внесли значимый вклад в подготовку статьи, прочли и одобрили финальную версию статьи перед публикацией.

Благодарности. Научно-техническая поддержка в подготовке рукописи и анализе данных для данной статьи выполнена группой MAG (Medical Advisors Group, http://mdwrt.com) в лице Якова Пахомова и Ильдара Хасанова. 


\section{СПИСОК ЛИТЕРАТУРЫ | REFERENCES}

1. Дедов И.И., Шестакова М.В., Майоров А.Ю., и др. Алгоритмы специализированной медицинской помощи больным сахарным диабетом. 9-й выпуск // Сахарный диабет. - 2019. - Т.22. №S1-1. - C. 1-144. [Dedov II, Shestakova MV, Mayorov AYu, at al. Standards of specialized diabetes care. Edited by Dedov II, Shestakova MV, Mayorov AYu. 9th edition. Diabetes Mellitus. 2019;22(S1-1):1-121. (In Russ).] doi: 10.14341/DM221S1.

2. Nauck MA, Ellis GC, Fleck PR, et al., Group AS 008. Efficacy and safety of adding the dipeptidyl peptidase-4 inhibitor alogliptin to metformin therapy in patients with type 2 diabetes inadequately controlled with metformin monotherapy: a multicentre, randomised, double-blind, placebo-controlled study. Int J Clin Pract. 2009;63(1):46-55. doi: 10.1111/j.1742-1241.2008.01933.x.

3. DeFronzo RA, Fleck PR, Wilson CA, Mekki Q; Alogliptin Study 010 Group. Efficacy and safety of the dipeptidyl peptidase-4 inhibitor alogliptin in patients with type 2 diabetes and inadequate glycemic control: a randomized, double-blind, placebo-controlled study. Diabetes Care. 2008;31(12):2315-2317. doi: 10.2337/dc08-1035.

4. Шестакова М.В., Качко В.А. ENTIRE: исследование реальной клинической практики применения алоглиптина в терапии пациентов с сахарным диабетом 2 типа в РФ // РМЖ. - 2019. T.27. - №8-1. - C. 3-10. [Shestakova MV, Kachko VA. ENTIRE: real clinical practice study on alogliptin application in the treatment of patients with type 2 diabetes mellitus in the Russian Federation. RMG. 2019;27(8-1):3-10. (In Russ).]

5. Eliasson B, Möller-Goede D, Eeg-Olofsson K, et al. Lowering of postprandial lipids in individuals with type 2 diabetes treated with alogliptin and/or pioglitazone: a randomised doubleblind placebo-controlled study. Diabetologia. 2012;55:915-925. doi: 10.1007/s00125-011-2447-3.

6. White WB, Cannon CP, Heller SR, et al. Alogliptin after Acute coronary syndrome in patients with type 2 diabetes. N Engl J Med. 2013;369(14):1327-1335. doi: 10.1056/NEJMoa1305889.

7. Home PD, Shen C, Hasan MI, et al. Predictive and explanatory factors of change in $\mathrm{HbA} 1 \mathrm{c}$ in a 24-week observational study of 66,726 people with type 2 diabetes starting insulin analogs. Diabetes Care. 2014;37(5):1237-1245. doi: 10.2337/dc13-2413.

8. Del Prato S, Camisasca R, Wilson C, Fleck P. Durability of the efficacy and safety of alogliptin compared with glipizide in type 2 diabetes mellitus: a 2-year study. Diabetes Obes Metab. 2014;16(12):1239-1246. doi: 10.1111/dom.12377.

9. Шестакова М.В. Опыт применения ситаглиптина (первого ингибитора ДПП-4) в лечении сахарного диабета 2 типа в Российской Федерации: результаты наблюдательной программы «Диа-Да» // Сахарный диабет. — 2010. — №3. C. 57-60. [Shestakova MV. Experience with sitagliptin (the first DPP-4 inhibitor) application to the treatment of type 2 diabetes mellitus in the Russian Federation: results of the Dia-Da observation program. Diabetes Mellitus. 2010;(3):57-60. (In Russ).]
10. Matthews DR, Paldánius PM, Proot P, et al. Glycaemic durability of an early combination therapy with vildagliptin and metformin versus sequential metformin monotherapy in newly diagnosed type 2 diabetes (VERIFY): a 5-year, multicentre, randomised, double-blind trial. Lancet. 2019;394(10208):1519-1529. doi: 10.1016/S01406736(19)32131-2.

11. Monami M, Vitale $V$, Ambrosio ML, et al. Effects on lipid profile of dipeptidyl peptidase 4 inhibitors, pioglitazone, acarbose, and sulfonylureas: meta-analysis of placebo-controlled trials. Adv Ther. 2012;29(9):736-746. doi: 10.1007/s12325-012-0045-5.

12. Mita T, Katakami N, Yoshii H, et al. Alogliptin, a dipeptidyl peptidase 4 inhibitor, prevents the progression of carotid atherosclerosis in patients with type 2 diabetes: the study of preventive effects of alogliptin on diabetic atherosclerosis (SPEAD-A). Diabetes Care. 2016;39(1):139-148. doi: 10.2337/dc15-0781.

13. Xiao C, Dash S, Morgantini C, et al. Sitagliptin, a DPP-4 inhibitor, acutely inhibits intestinal lipoprotein particle secretion in healthy humans. Diabetes. 2014;63(7):2394-2401. doi: 10.2337/db13-1654.

14. Nauck MA, Meier JJ, Cavender MA, et al. Cardiovascular actions and clinical outcomes with glucagon-like peptide-1 receptor agonists and dipeptidyl peptidase-4 inhibitors. Circulation. 2017;136(9):849-870. doi: 10.1161/CIRCULATIONAHA.117.028136.

15. Ogawa S, Ishiki M, Nako K, et al. Sitagliptin, a dipeptidyl peptidase-4 inhibitor, decreases systolic blood pressure in Japanese hypertensive patients with type 2 diabetes. Tohoku J Exp Med. 2011;223(2):133-135. doi: 10.1620/tjem.223.133.

16. Duvnjak L, Blaslov K. Dipeptidyl peptidase-4 inhibitors improve arterial stiffness, blood pressure, lipid profile and inflammation parameters in patients with type 2 diabetes mellitus. Diabetol Metab Syndr. 2016;8:26. doi: 10.1186/s13098-016-0144-6.

17. Evans M, Schweizer A, Foley JE. Blood pressure and fasting lipid changes after 24 weeks' treatment with vildagliptin: a pooled analysis in $>2,000$ previously drug-naive patients with type 2 diabetes mellitus. Vasc Health Risk Manag. 2016;12:337-340. doi: 10.2147NHRM.S112148.

18. Chen K, Zhuo T, Wang J, Mei Q. Saxagliptin upregulates nesfatin-1 secretion and ameliorates insulin resistance and metabolic profiles in type 2 diabetes mellitus. Metab Syndr Relat Disord. 2018;16(7):336-341. doi: 10.1089/met.2018.0010.

19. Yuasa S, Sato K, Furuki T, et al. Primary care-based investigation of the effect of sitagliptin on blood pressure in hypertensive patients with type 2 diabetes. J Clin Med Res. 2017;9(3):188-192. doi: 10.14740/jocmr2820w.

20. Mason RP, Jacob RF, Kubant R, et al. Dipeptidyl peptidase-4 inhibition with saxagliptin enhanced nitric oxide release and reduced blood pressure and sICAM-1 levels in hypertensive rats. J CardiovasC Pharmacol. 2012;60(5):467-473. doi: 10.1097/FJC.0b013e31826be204.

21. Zhang J, Chen Q, Zhong J, et al. DPP-4 Inhibitors as potential candidates for anti-hypersensitive therapy: improving vascular inflammation and assisting the action of traditional antihypertensive drugs. Front Immunol. 2019;10:1050. doi: 10.3389/fimmu.2019.01050.

Рукопись получена: 14.01.2020. Одобрена к публикации: 29.05.2020. Опубликована online: 28.07.2020.

ИНФОРМАЦИЯ ОБ АВТОРАХ [AUTHORS INFO]

Шестакова Марина Владимировна, д.м.н., профессор, академик РАH [Marina V. Shestakova, MD, PhD, Professor]; адрес: Россия, 117036, Москва, ул. Дм. Ульянова, д. 11 [address: 11 Dm.Ulyanova street, 117036 Moscow, Russia]; ORCID: https://orcid.org/0000-0002-5057-127X; eLibrary SPIN: 7584-7015; e-mail: nephro@endocrincentr.ru

Шестакова Екатерина Анатольевна, к.м.H. [Ekaterina A. Shestakova, MD, PhD];

ORCID: http://orcid.org/0000-0001-6612-6851; eLibrary SPIN: 1124-7600; e-mail: katiashestakova@mail.ru

Качко Вера Александровна [Vera A. Kachko, MD]; ORCID: http://orcid.org/0000-0002-0617-7312;

eLibrary SPIN: 5869-7470; e-mail: Vera.Kachko@takeda.com

\section{ЦИТИРОВАТЬ:}

Шестакова М.В., Шестакова Е.А., Качко В.А. Особенности применения алоглиптина у различных групп пациентов с сахарным диабетом 2 типа: дополнительные результаты исследования ENTIRE// Проблемы эндокринологии. — 2020. — T. 66. — №2. - C. 49-60. doi: https://doi.org/10.14341/probl12273 


\section{TO CITE THIS ARTICLE:}

Shestakova MV, Shestakova EA, Kachko VA. Specific features of the use of Alogliptin in various groups of patients with type 2 diabetes mellitus: additional results of the ENTIRE study. Problems of Endocrinology. 2020;66(2):49-60. doi: https://doi.org/10.14341/probl12273 